\title{
La Silla-QUEST Variability Survey in the Southern Hemisphere
}

\author{
Ellie Hadjiyska ${ }^{1}$, David Rabinowitz ${ }^{1}$, Charles Baltay ${ }^{1}$, \\ Nancy Ellman ${ }^{1}$, Peter Nugent ${ }^{2}$, Robert Zinn ${ }^{3}$, Benjamin Horowitz ${ }^{1}$, \\ Ryan McKinnon ${ }^{1}$, Lissa R. Miller ${ }^{3}$ \\ ${ }^{1}$ Center for Astronomy \& Astrophysics, Yale University, New Haven, CT 06520-8120 USA \\ email: ellie.hadjiyska@yale.edu \\ ${ }^{2}$ Lawrence Berkeley National Laboratory, Berkeley, CA, 94720-8139 USA \\ ${ }^{3}$ Dept. of Astronomy, Yale University, \\ P.O. Box 208101, New Haven, CT 06520-8101 USA
}

\begin{abstract}
We describe the La Silla-QUEST (LSQ) Variability Survey. LSQ is a dedicated wide-field synoptic survey in the Southern Hemisphere, focussing on the discovery and study of transients ranging from low redshift $(\mathrm{z}<0.1)$ SN Ia, Tidal Disruption events, RR Lyræ variables, CVs, Quasars, TNOs and others. The survey utilizes the 1.0-m Schmidt Telescope of the European Southern Observatory at La Silla, Chile, with the large-area QUEST camera, a mosaic of 112 CCDs with field of view of 9.6 square degrees. The LSQ Survey was commissioned in 2009 , and is now regularly covering $\sim 1000$ square deg per night with a repeat cadence of hours to days. The data are currently processed on a daily basis. We present here a first look at the photometric capabilities of LSQ and we discuss some of the most interesting recent transient detections.
\end{abstract}

Keywords. surveys, techniques: photometric, supernovae: Type Ia, stars: variables

\section{Introduction}

After the completion of the Palomar-QUEST northern sky survey in September 2008 the QUEST Large Field Camera (Baltay et al. 2007) was moved and installed on the 1.0-m ESO Schmidt in La Silla and had first light on April 24, 2009. Since September 2009 the southern survey has been routinely observed (Andrews et al. 2008), and the telescope and camera are controlled from Yale and are fully robotic. We have $90 \%$ of the time on the telescope with $10 \%$ allocated to Chile. The QUEST camera consists of 112 CCDs of $600 \times 2400$ Sarnoff thinned pixels, back illuminated devices with $13 \mu \mathrm{m}$ $\times 13 \mu \mathrm{m}$ pixel pitch. The camera covers an area of $4^{\circ} .6 \times 3^{\circ} .6$ on the sky and a plate scale of $0.86 \mathrm{arcsec} / \mathrm{pixel}$. The survey covers $\sim 1000$ square degrees per night, primarily between $\pm 25^{\circ}$ to allow for follow-up from both hemispheres. The LSQ variability (SN and transient) survey uses 60-sec exposures (and the TNO survey $180 \mathrm{sec}$ ) taken twice a night with a cadence of 2 nights in one broad-band filter of 4000 to $7000 \AA$ (Qst*-band). The seeing at La Silla for the 60 -sec exposures is 1.7 arcsec FWHM, reaching a depth of 20.5 mag. The LSQ survey subtraction pipeline has started producing between 400 and 900 transient candidates each night (Fig. 1). 

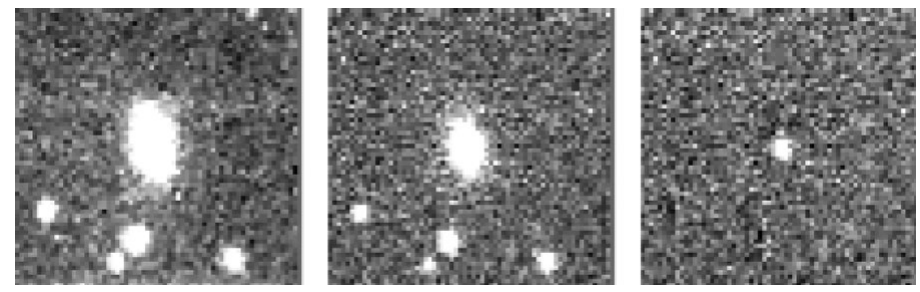

Figure 1. A possible supernova. From left - reference image, night1, subtracted image.

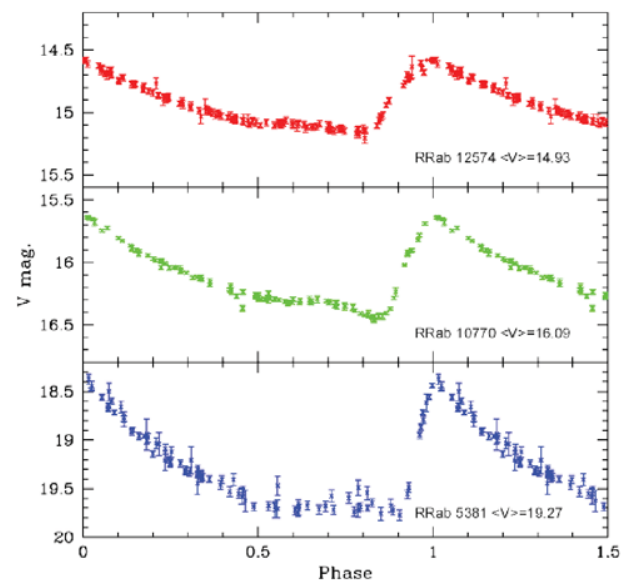

(a) RR Lyrae V magnitude light curves for RRab 12574, 10770 and 5381.

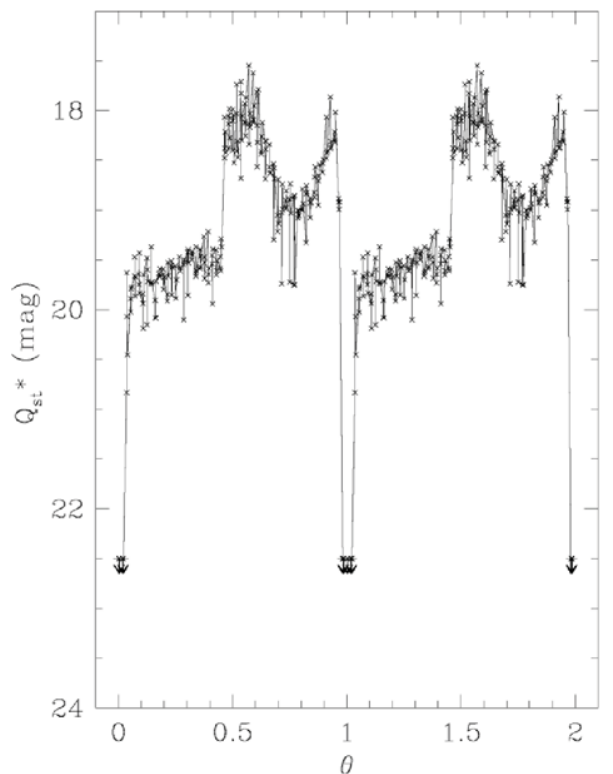

(b) Relative Qst*-band brightness versus orbital phase $\theta$, from LSQ observations.

Figure 2. LSQ Example Transient Detections.

\section{Transient Detections}

\subsection{RR Lyrce Variables}

The LSQ RR Lyræ star (RRLS) survey is searching the galactic halo for RRLS that have $V$ magnitudes between roughly 14 and 20. Because RRLS are excellent standard candles, they provide a powerful probe of the density distribution of the halo, which is being examined for halo substructure. Plotted are the $V$ magnitude light-curves for three type ab RRLS (Fig. 2a), which illustrate the typical photometric precisions at these magnitudes. From their mean $V$ magnitudes, we estimate that RRab 12574, 10770, and 5381 lie 7,13 , and $52 \mathrm{kpc}$ from the Sun, respectively.

\subsection{A Deep Eclipsing $C V$}

A deep eclipsing cataclysmic variable (Rabinowitz et al. 2011a) was discovered with eclipse depths $>5.7$ magnitudes, orbital period $94.657 \mathrm{~min}$, and peak brightness $V \sim 18$ at J2000 position $17 \mathrm{~h} 25 \mathrm{~m} 54.8 \mathrm{~s},-64 \mathrm{deg} 38 \mathrm{~min} 39 \mathrm{sec}$. Light curves in $B, V, R, I, z$ and $J$ were obtained with SMARTS $1.3-\mathrm{m}$ and $1.0-\mathrm{m}$ telescopes at Cerro Tololo and spectra from 3500 to $9000 \AA$ with the SOAR 4.3-m telescope at Cerro Pachon. The optical light curves (Fig. 2b) show a deep, 5-min eclipse immediately followed by a shallow 38-min eclipse and then sinusoidal variation. No eclipses appear in $J$. During the deep eclipse 

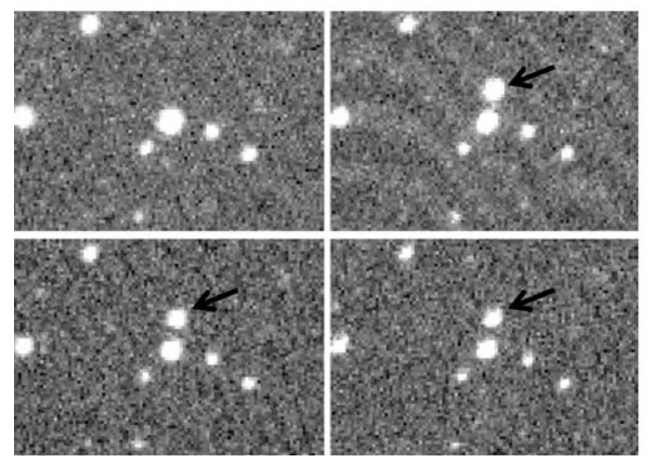

(a) Before and after discovery images of the dwarf novae.

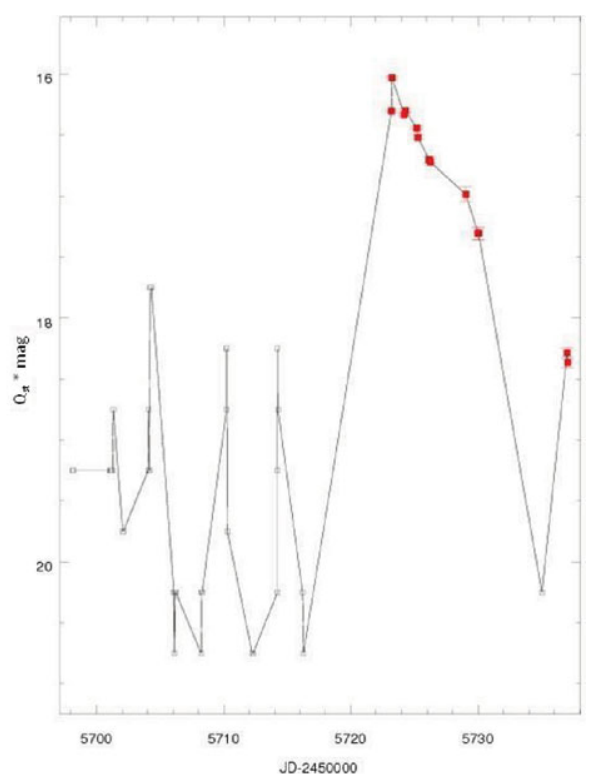

(b) Relative Qst* light curve of LSQ J16531857-1617542, upper limits and error barred detections.

Figure 3. The first followed up LSQ subtraction pipeline transient candidate.

the measure of $V-J>7.1$ corresponds to a spectral type M8 or later secondary. The spectra show strong Hydrogen emission lines, Doppler broadened by $600-1300 \mathrm{~km} \mathrm{~s}-1$, oscillating with radial velocity that peaks at mid deep eclipse with semi-amplitude $500 \pm$ $22 \mathrm{~km} \mathrm{~s}^{-1}$. It is suggested that LSQ172554.8-643839 is a polar with a low-mass secondary viewed at high inclination.

\subsection{A Dwarf Nova}

An apparent dwarf nova was discovered (Rabinowitz et al. 2011b) on June 11.046 (when the variable was at magnitude $R=16.3$ ) and June 11.063 UT (at R $=16.0$ ) (Figs. 3a and $3 \mathrm{~b}$ ). A faint source is reported at this position in the Guide Star Catalog V2.3.2 (with $B \mathrm{j}=20.76)$. Simultaneous visible and $J$-band observations were taken with ANDICAM on the 1.3-m SMARTS telescope at Cerro Tololo. A spectrum (range 0.350-0.966 nm) taken on June 26 with GMOS on the Gemini South telescope reveals strong H- $\alpha$ and $\mathrm{H}-\beta$ emission lines, with H-alpha clearly double-peaked, indicating the presence of an accretion disk with a rotational velocity of $1000 \mathrm{~km} \mathrm{~s}^{-1}$.

This work has been made possible with the help of NERSC computer resources and funding from DOE and NASA.

\section{References}

Andrews, P., et al. 2008, PASP, 120, 703A

Baltay, C., et al. 2007, PASP, 119, 1278B

Rabinowitz, D., et al. 2011, ApJ, 732, 51

Rabinowitz, D., Baltay, C., Ellman, N., Hadjiyska, E., \& Tourtellote, S. 2011, CBET, 2757, 1R 